\title{
MSC-Derived Extracellular Vesicles to Heal Diabetic Wounds: a Systematic Review and Meta-Analysis of Preclinical Animal Studies
}

\author{
Adrian J. M. Bailey ${ }^{1,2,3} \cdot$ Heidi Li $^{1} \cdot$ Aidan M. Kirkham ${ }^{2,3} \cdot$ Alvin Tieu $^{1,3,4,5} \cdot$ Harinad B. Maganti ${ }^{2,3} \cdot$ Risa Shorr $^{6}$. \\ Dean A. Fergusson ${ }^{1,3,7,8} \cdot$ Manoj M. Lalu ${ }^{1,3,4,5,9} \cdot$ Heidi Elomazzen ${ }^{2} \cdot$ David S. Allan $^{1,2,3,4,7,10}$
}

Accepted: 29 March 2021 / Published online: 24 April 2021

(C) The Author(s), under exclusive licence to Springer Science+Business Media, LLC, part of Springer Nature 2021

\begin{abstract}
Introduction Extracellular vesicles from mesenchymal stromal cells (MSC-EVs) have shown promise in wound healing. Their use in diabetic wounds review of preclinical studies is needed to determine the efficacy of MSC-EVs in the treatment of diabetic wounds to accelerate the clinical translation of this cell-based therapy.

Methods PubMed and Embase were searched (to June 23, 2020). All English-language, full-text, controlled interventional studies comparing MSC-EVs to placebo or a "no treatment" arm in animal models of diabetic wounds were included. Study outcomes, including wound closure (primary outcome), scar width, blood vessel number and density, and re-epithelialisation were pooled using a random effects meta-analysis. Risk of bias (ROB) was assessed using the SYRCLE tool for pre-clinical animal studies.

Results A total of 313 unique records were identified from our search, with 10 full text articles satisfying inclusion criteria $(n=$ 136 animals). The administration of MSC-EVs improved closure of diabetic wounds compared to controls with a large observed effect (Standardized Mean Difference (SMD) 5.48, 95\% Confidence Interval (CI) 3.55-8.13). Healing was further enhanced using MSC-EVs enriched in non-coding RNAs or microRNAs compared to controls (SMD 9.89, 95\%CI 7.32-12.46). Other outcomes, such as blood vessel density and number, scar width, and re-epithelialisation were improved with the administration of MSC-EVs, with a large effect. ROB across studies was unclear.

Conclusion MSC-EVs, particularly following enrichment for specific RNAs, are a promising treatment for diabetic wounds in pre-clinical studies and translation to the clinical domain appears warranted.

Registration PROSPERO \#CRD42020199327 [248].
\end{abstract}

Keywords Mesenchymal stromal cells $\cdot$ Extracellular vesicles $\cdot$ Exosomes $\cdot$ Diabetes $\cdot$ Wound healing

\section{Introduction}

Chronic foot ulcers and delayed wound healing are debilitating complications of diabetes mellitus [1] that increase patient

David S. Allan

daallan@toh.ca

1 Faculty of Medicine, University of Ottawa, Ottawa, Ontario, Canada

2 Stem Cells and Centre for Innovation, Canadian Blood Services, Ottawa, Canada

3 Clinical Epidemiology, Ottawa Hospital Research Institute, Ottawa, Canada

4 Regenerative Medicine Programs, Ottawa Hospital Research Institute, Ottawa, Canada

5 Departments of Cellular and Molecular Medicine, University of Ottawa, Ottawa, Canada risk of gangrene, amputation, disability, and death $[2,3]$. Effective management of diabetic complications will be increasingly needed, as the prevalence of diabetes mellitus is expected to rise from approximately $6.4 \%$ in 2010 to $7.7 \%$

6 Library and Information Services, The Ottawa Hospital, Ottawa, Canada

7 Medicine, The Ottawa Hospital, University of Ottawa, Ottawa, Canada

8 School of Public Health and Epidemiology, University of Ottawa, Ottawa, Canada

9 Departments of Anesthesiology and Pain Medicine, University of Ottawa, Ottawa, Canada

10 Biochemistry, Microbiology \& Immunology, University of Ottawa, Ottawa, Canada 
by 2030 [4]. Unfortunately, current therapies for diabetic wounds remain insufficient [3], and healing is often prolonged due to poor perfusion and chronic inflammation [5].

Mesenchymal stromal cells (MSCs) have been investigated extensively for their role in tissue repair due to their angiogenic and immune modulatory properties [6-9]. MSCs contribute to angiogenesis $[10,11]$ by increasing capillary growth and increasing angiogenic factors in burns [12, 13], pressure ulcers, and diabetic wounds [14, 15]. In clinical trials, MSCs have demonstrated efficacy for the treatment of several immune conditions such as chronic ulcers in leprosy [16], rheumatoid arthritis [17], Crohn's disease [18], graft versus host disease [19], and eczema [20]. Moreover, MSCs have been studied in a broad range of immunological conditions [21], have been deemed largely safe across a wide range of diseases [22] and continue to be studied for many other indications [23]. A significant number of registered clinical trials of MSCs and/or derivative therapies such as conditioned media or extracellular vesicles from MSCs are currently enrolling patients to study the efficacy in wound healing in patients with burns, diabetes, and other predisposing diseases (see clinicaltrials.gov, searched March 9, 2021). Notably, MSCs are approved in Europe for the treatment of perianal fistulas secondary to Crohn's disease [24]. Additionally, MSCs can promote wound healing in a variety of pre-clinical models and clinical studies, including diabetic wounds $[25,26]$. In particular, initial clinical reports suggest that patients with skin ulcers receiving MSCs experience faster wound closure rates and less pain compared to conventional therapy $[8,25]$.

Despite the emerging promise of MSC-based therapies, concerns about ectopic tissue formation after administration and challenges related to storage and cost have dampened enthusiasm. A cell-free alternative, such as MSC-derived extracellular vesicles (MSC-EVs) represents an appealing therapy to harness the advantages of MSC-based therapies and provide an "off-the-shelf" treatment alternative.

EVs are nano-sized vesicles secreted by many cell types, including MSCs, and are classified as small EVs $(\sim 30-$ $150 \mathrm{~nm}$ ), which includes exosomes that are released from endosomal-derived multivesicular bodies after merging with the plasma membrane, and large EVs ( 150-1000 nm) which includes microvesicles that are formed by blebbing of the plasma membrane [27]. EVs can travel and merge with the plasma membrane of target cells and release micro RNAs, proteins, and other ligands that can alter signalling pathways and facilitate cell to cell communication in target cells, including the activation of angiogenic pathways critical for tissue repair and recovery. Increasing evidence suggests that MSCEVs share similar angiogenic, immune modulatory, and therapeutic properties to MSCs [28] and likely account for much of the paracrine effects of MSC-based therapies [29-31]. Specifically, a previous mechanistic analysis of all studies investigating MSC-EVs in pre-clinical models of disease demonstrated that angiogenesis and inflammation are some of the most commonly targeted pathways by MSC-EVs [32].

Although MSC-EVs have shown efficacy in a variety of wound healing applications, the evidence for the use of MSCEVs in diabetic wound healing remains pre-clinical and uncertain. A systematic review and meta-analysis are needed to determine the efficacy of MSC-EVs in the treatment of diabetic wounds to accelerate and optimize the translation towards clinical investigation.

\section{Methods}

This study's protocol was registered on PROSPERO (CRD42020199327, September 10, 2020). This report was prepared in accordance with the PRISMA checklist [33] (see Supplemental Checklist).

\section{Eligibility Criteria}

Eligibility criteria were developed a priori to determine which full-text articles would be eligible for inclusion in this systematic review and meta-analysis. Study population, intervention, comparator, outcome, and study design (PICOS) parameters were predefined to allow for objective, reproducible analysis, as is standard for systematic reviews.

\section{Population}

Any in vivo animal model of diabetic wounds was included. Studies involving in vitro, ex vivo, or invertebrate pre-clinical models of diabetic wounds were excluded.

\section{Intervention}

Studies investigating the use of EVs (e.g., small or large EVs, exosomes, and/or microvesicles) isolated from MSCs expanded from any tissue source for the treatment of diabetic wounds were included. Any xenogeneic, allogeneic, or autologous administration of MSC-EVs to diabetic wounds was permitted, including the administration of MSC-EVs via a hydrogel. Unmodified, preconditioned, modified (e.g., gene transfected, protein or microRNA overexpression) MSC-EVs were included. Administration of MSC-EVs as a co-treatment with another therapy was permissible. Studies investigating the effect of MSCs or that used EVs from other sources were excluded.

\section{Comparator}

Any type of comparator was included (e.g., placebo, vehicle control, MSCs, hydrogels, fibroblasts, fibroblast EVs, etc.). Non-comparative studies were excluded. 


\section{Outcome}

The primary outcome of this study was wound closure. All studies were included, regardless of initial wound size or method of wound size measurement. Secondary outcomes included blood vessel density, blood vessel number, collagen deposition, inflammation, scar width, side-effects, and adverse events. As the focus of this study was on in vivo outcomes, in vitro outcomes were excluded.

\section{Study Design}

All English-language, full-text, controlled interventional studies (randomized, quasi-randomized, and non-randomized) comparing MSC-EVs to placebo or a "no treatment" arm in animal models of diabetic wounds were included. Review articles, non-comparative studies, commentaries, editorials, care reports, case-series, and other study types were excluded.

\section{Literature Search Strategy}

A comprehensive literature search strategy was developed in collaboration with a medical librarian (RS) specializing in systematic review searches and was peer reviewed [34]. The search strategy was used to search MEDLINE and EMBASE from inception to June 23rd, 2020. The reference lists of included studies and any relevant reviews captured by the search strategy were reviewed to ensure that all relevant articles were captured. The full search strategy is outlined in Table S1.

\section{Study Selection Process}

All identified citations were imported into Rayyan (https:// rayyan.qcri.org/) for management of search records, after removing duplicates. Study titles and abstracts were screened by two independent reviewers in duplicate (AB and $A K$ ), and the full texts of any potentially relevant studies were screened to determine final eligibility. In cases of disagreement between reviewers, consensus was achieved through discussion with a third team member (DA). The study selection process was summarized using a flow diagram, as per PRISMA recommendations.

\section{Data Extraction}

Relevant data was extracted by two independent reviewers (AB, HL) from included studies using a standardized and pilot-tested data extraction form in Excel (Microsoft, Seattle, USA). In studies where the raw data were not presented, the data was extracted from figures using Digitizelt (Version 2.2; Braunschweig, Germany). Data regarding study characteristics (e.g. authors, publication year, country of study), study populations (e.g. species, strain, diabetic model, wound size, depth), intervention characteristics (e.g. whether all of the International Society for Cellular Therapy (ISCT) minimal criteria for MSC characterization [35] were met, MSC source, MSC-EV dose, route of administration), study design (e.g. comparator, sample size, MSC-EV methods of isolation and characterization), outcomes (e.g. wound closure, blood vessel density, collagen deposition, scar width), adverse events, and details concerning risk of bias were extracted.

\section{Risk of Bias}

Two independent reviewers (AB, HL) assessed the risk of bias of each included study according to the Systematic Review Centre for Laboratory animal Experimentation (SYRCLE) risk of bias tool for animal studies [36]. Specifically, selection bias (sequence generation, baseline characteristics, allocation concealment), performance bias (random housing, blinding of investigators), detection bias (random outcome assessment, blinding of assessors), attrition bias (incomplete outcome data), and reporting bias (selective outcome reporting) were assessed. No other sources of bias were investigated.

\section{Data Analysis}

Study results were pooled using Review Manager 5.3 (Cochrane) [37]. For continuous outcomes, either a mean difference (MD) or a standardized mean difference (SMD) was calculated using random effects meta-analyses. SMD was selected for outcomes that were likely to be affected by variability of the initial wound size across studies, such as wound closure, blood vessel number, wound length, and scar width [38]. MD was selected for outcomes unlikely to be affected by variability in initial wound size, such as blood vessel density [38]. Since time points across studies were heterogeneous, the maximum effect estimate for each study, between 7 and 14 days, was used in the pooled analysis [39]. Only one study reported a maximum effect beyond 14 days but also provided data for 7 and 14 days [40]. All data is presented with $95 \%$ confidence intervals (CI). A meta-analysis was only performed when two or more studies reported on the same outcome. Any study that did not provide adequate data for inclusion in a meta-analysis was analyzed descriptively. Any shared control groups were accounted for in the metaanalysis by dividing the control group sample size by the number of experimental groups. The $\mathrm{I}^{2}$ statistic was used to assess statistical heterogeneity [38]. Subgroup analyses were determined a priori in our study protocol and were conducted to determine whether the effect of MSC-EVs varied according to the use of a hydrogel or the genetic modification of MSCEVs. Considering the number of studies included in our quantitative analyses $(n=10)$, scatter plot asymmetry and statistical tests for publication bias were not performed, although 
mentioned in our PROSPERO protocol. Finally, $p<0.05$ was considered significant for all analyses.

\section{Results}

A total of 313 unique records were identified from our search. After screening titles and abstracts for potential relevance, 16 full text articles were retrieved and underwent full review according to the inclusion and exclusion criteria. Six articles were excluded for various reasons: 3 studies isolated cells from cells other than MSCs, 1 study did not administer EVs, 1 study of wounds did not include a diabetic aspect to the animal model, and 1 study was in Chinese (Fig. 1). In total, 10 studies [40-49] addressed the therapeutic use of MSC-EVs in diabetic wound healing and were included for qualitative analysis. Nine studies were included in our quantitative metaanalysis (Fig. 1).

\section{Study Characteristics}

Characteristics of the 10 included studies are summarized in Table 1. Study publication date ranged from 2015 to 2020 and studies were conducted in China $(n=9)$ and $\operatorname{Iran}(n=1)$. The studies used rats $(n=5)$ and mice $(n=5)$. The method used to induce diabetes was either chemical (streptozotocin, $n=9$ ) or transgenic $(n=1)$. A circular cutaneous wound was used for injury in all studies and wound diameter ranged from 8 to $20 \mathrm{~mm}$. Eighty percent $(n=8)$ of the studies reported fullthickness wounds.

All studies used MSCs that satisfied the ISCT criteria [35] of adherence to plastic and multipotent differentiation potential. Only one study reported sufficient cell surface marker expression to fully meet ISCT criteria [44]. Several studies $(n=4)$ did not report positive expression of one or more markers $[42,45,46,48]$ and other $(n=8)$ failed to report the absence of one or more markers [40, 41, 43, 45-49] included in the ISCT criteria. MSCs were derived from human tissue sources in 8 studies, including synovia $(n=2)$, bone marrow $(n=1)$, adipose tissue $(\mathrm{n}=1)$, umbilical cord $(\mathrm{n}=1)$, menstrual blood $(n=1)$, decidua $(n=1)$, and gingival tissue $(n=1)$ and from non-human tissue sources in 2 studies, including mouse bone marrow $(\mathrm{n}=1)$ and adipose tissue $(n=1)$. EVs were isolated either using ultracentrifugation $(n=9)$ or a size exclusion column $(\mathrm{n}=1)$. The most common method of MSC-EV characterisation was electron microscopy $(n=10)$, followed by Western blot (various combinations of Alix, CD9, CD63, CD81, TSG101, HSP 70, GM 130, and Grp94) $(\mathrm{n}=9)$, dynamic light scattering $(n=3)$, nanoparticle tracking analysis $(n=2)$, and flow cytometry (for non-EV surface markers CD73, CD90, CD105 to confirm MSC origin) (n = 1). Doses of MSC-EVs varied from approximately 10 to $200 \mu \mathrm{g}$ (reported in 6 studies) or $1.83 \times 10^{10}$ to $5.22 \times 10^{10}$ particles (reported in 2 studies; see Table 1), with most studies $(n=6)$ administering MSC-EVs as a single dose after the establishment of a diabetic wound. The dose of MSC-EVs was not reported in two studies [44, 45]. Across studies, MSC-EVs were delivered after embedding in hydrogel material $(n=4)$, via intradermal injections $(n=2)$, direct injection into the wound $(n=2)$, or by subcutaneous injections $(n=2)$. The size of administered EVs was reported as $<150 \mathrm{~nm}$ in 7 studies, between 40 and $200 \mathrm{~nm}$ [42], or as a mean size of $85 \pm 36 \mathrm{~nm}$ [45] or $127 \pm 56 \mathrm{~nm}$ [47].

Three studies administered MSC-EVs that were enriched for specific micro or circular RNAs that target mechanisms involved in chronic wound establishment. One study [46] enriched their MSC-EVs with the circular RNA molecule, mmu_circ_0000250, to target miR-128-3p, which has been shown to regulate SIRT1 expression, a regulator of inflammation. Another study [48] enriched MSC-EVs with miR126 , which has been implicated in improved angiogenesis in response to ischemia via the phosphatidylinositol 3kinase (PI3K)/AKT and mitogen-activated protein kinase (MAPK)/extracellular signal-regulated kinase (ERK) pathways. A third study [44] used genetically modified MSCs to generate EVs that were enriched for long non-coding RNA H19 (lnc H19), which has a putative role in PTEN expression, leading to the regulation of the PI3K/AKT signaling pathway and suppression of inflammatory responses and apoptosis.

\section{Primary Outcome - Wound Closure}

Eight studies reported data on wound closure of diabetic wounds with and without treatment with MSC-EVs. The administration of MSC-EVs significantly increased wound closure of diabetic wounds compared to controls (SMD 5.84, 95\% CI 3.55 to $8.13, P<0.001, \mathrm{I}^{2}=82 \%$ ) (Fig. 2). In subgroup analysis, MSC-EVs embedded in a hydrogel was associated with improved wound closure compared to controls (4 studies, SMD 7.45, 95\% CI 5.04-9.82; Fig. 3), but the effect was not significantly different compared to the use of MSC-EVs administered without a hydrogel ( 7 intervention groups from 5 studies, SMD 4.97, 95\% CI 2.34-7.60; $p=0.17$; Fig. 3). In contrast, the improvement in diabetic wound closure observed after the administration of MSC-EVs enriched for specific RNAs (3 studies; SMD 9.89, 95\% CI 7.32 to 12.46) was significantly greater compared to the improvement associated with unmodified MSC-EVs (8 intervention cohorts in 6 studies; SMD 4.30, 95\% CI 2.25-6.35; $p=0.0009$ ) (Fig. 4). One study directly compared modified MSC-EVs to unmodified control MSC-EVs and found improved wound closure with modified MSC-EVs compared to unmodified MSC-EVs [44]. 
Fig. 1 PRISMA flow diagram for study selection process. PubMed, Embase, and Cochrane register of controlled trials were searched from inception to June 22nd, 2020
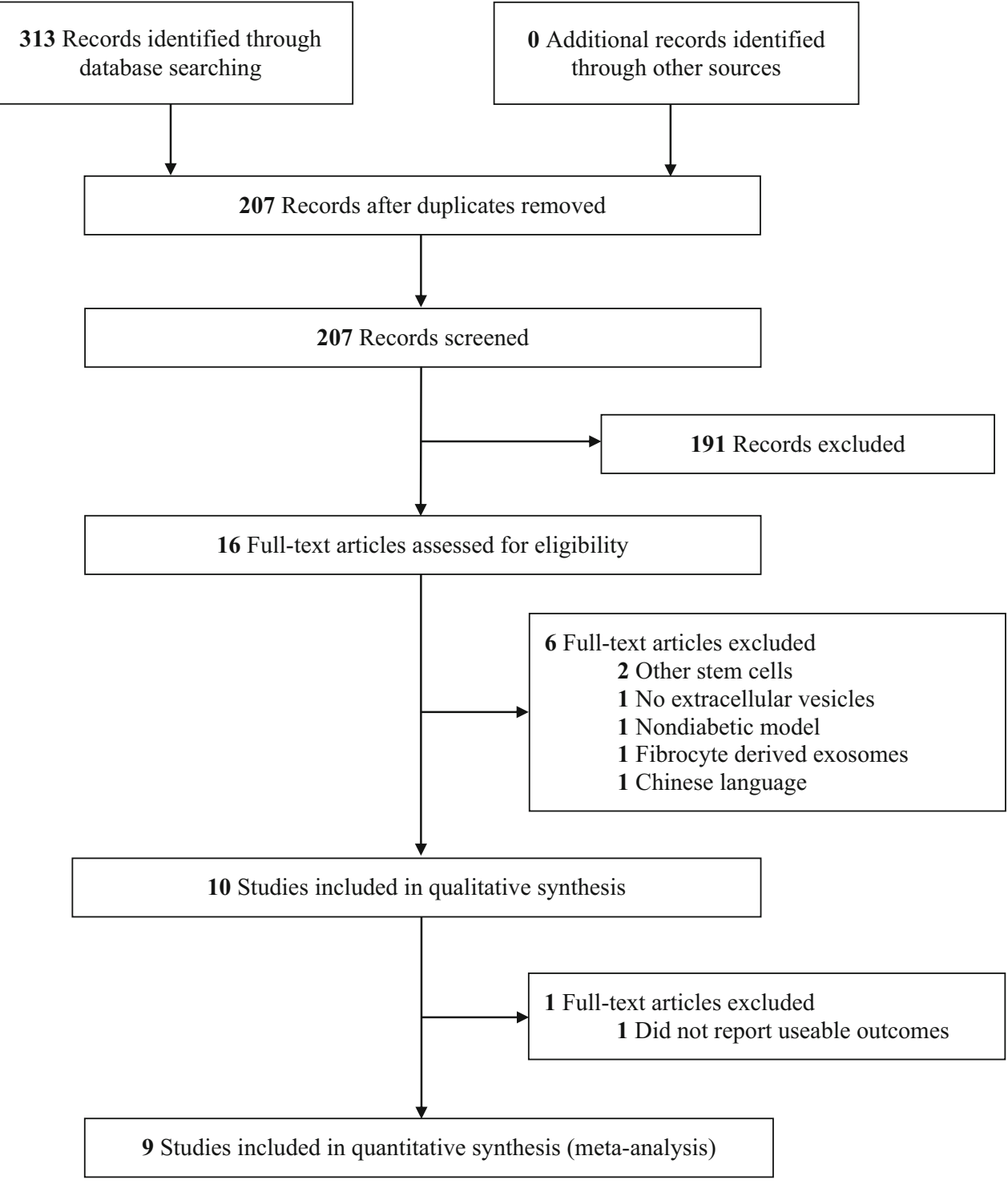

\section{Secondary Outcomes}

\section{Blood Vessel Density and Number}

Three studies reported blood vessel density, all of which demonstrated benefit with the use of MSC-EVs. Across studies, the administration of MSC-EVs was associated with significantly greater blood vessel density compared to controls (mean difference of 24.2 blood vessels per $\mathrm{mm}^{2}, 95 \% \mathrm{CI}$ 22.3 to $26.0, P<0.001, \mathrm{I}^{2}=0 \%$ ) (Fig. S1). Five studies reported blood vessel number, with four reporting an increase with MSC-EV treatment. Pooled analysis demonstrated that administration of MSC-EVs was associated with a significantly greater number of blood vessels compared to controls (SMD $4.84,95 \%$ CI 2.52 to $7.17, \mathrm{P}<0.001, \mathrm{I}^{2}=67 \%$ ) (Fig. S2). Two studies investigated the expression levels of angiogenic factors. One study reported that VEGFa was significantly upregulated in wounds receiving MSC-EVs compared to controls [42]. The other study that reported on angiogenic factors noted that MSC-EVs enriched for long noncoding RNA H19 significantly increased expression of VEGF, TGF- $\beta 1$, and $\alpha$ SMA in wounds compared to controls [44]. Lastly, one study demonstrated that miR-126 knockdown decreased tubule and branching formation in vitro [43].

\section{Re-Epithelialization}

Four studies reported increased re-epithelialisation of diabetic wounds with MSC-EVs, all of which demonstrated benefit. The administration of MSC-EVs significantly increased reepithelialisation of diabetic wounds compared to controls, with a large effect (SMD 6.20, 95\% CI 4.45 to 7.94, $\left.P<0.001, \mathrm{I}^{2}=0 \%\right)($ Fig. S3). 


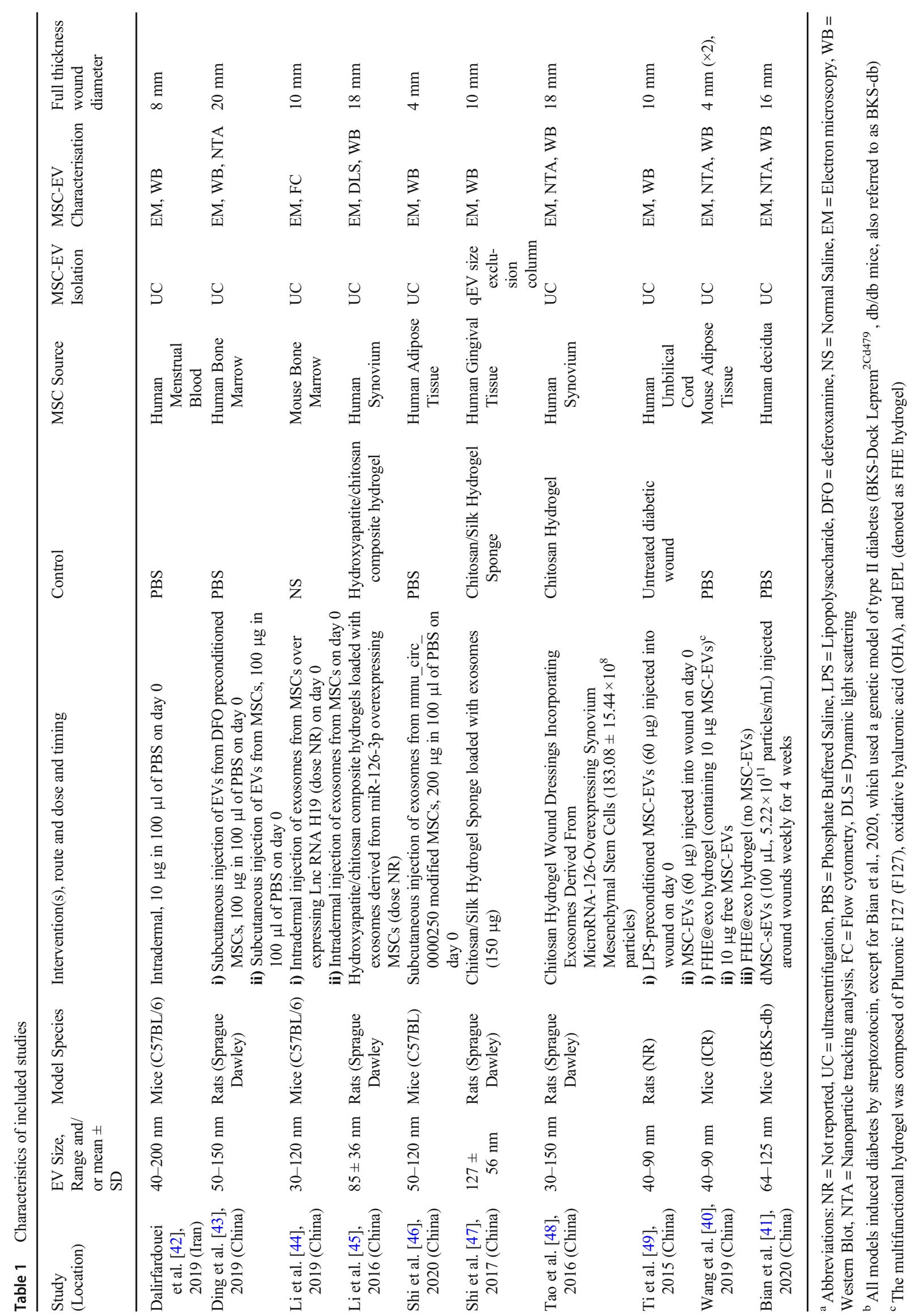




\section{Collagen Deposition}

Six studies investigated collagen deposition in diabetic wounds with the administration of MSC-EVs, all of which showed increase collagen deposition and/or maturity. Across studies, increased deposition of collagen fibers [41, 45, 47, 48], specifically Type I and Type III collagen [40], collagen maturity $[45,48]$, and collagen organisation [41] were reported in diabetic wounds receiving MSC-EVs compared to controls. Studies also reported increased expression of collagen related genes, such as increased Collagen I expression [41, 44] and Col1:Col3 expression ratio [42], in diabetic wounds receiving MSC-EVs compared to controls. This effect was increased in studies with the administration of the MSC-EVs embedded within a hydrogel $[47,48,40]$ or with the administration of MSC-EVs enriched in miRNA-126 [48].

\section{Scar Width}

Four studies reported on the degree of wound scarring, all of which demonstrated benefit. The administration of MSC-EVs was associated with a significantly smaller scar width compared to controls, with a large effect (SMD 4.60, 95\% CI 3.12 to $6.08, P<0.001, \mathrm{I}^{2}=0 \%$ ) (Fig. S4). These findings were corroborated by the studies that described scarring descriptively $[46,40]$.

\section{Inflammation}

Across five studies, inflammatory markers were decreased in diabetic wounds receiving MSC-EVs compared to controls, however, a meta-analysis was not possible due to the heterogeneity in reported outcomes. In particular, studies reported decreased levels of IL- $1 \beta$ and TNF- $\alpha$ [44], Toll-like receptor 4 and p-65 expression [49], and Rela gene expression [42]. Studies also reported decreased apoptosis rate [46] and increased expression of genes associated with cell proliferation, such as PTEN [44] and SIRT1 and LC3 expression [46] in MSC-EV treated wounds. Studies reported reduced inflammatory cell infiltration [49] and increased M2 macrophage polarisation $[42,49]$ in diabetic wounds treated with MSCEVs. Lastly, one study found that the knockdown of AKT abolished the immune modulatory effect of EVs from LPS pre-conditioned MSCs [49].

\section{Adverse Events}

One study reported that there were not any adverse events observed in any animal [45]. The presence or absence of adverse events or complications due to MSC-EV treatment in the remaining studies was unclear, as this information was not reported. None of the studies reported any dropouts or mortality of animals in any group.

\section{Risk of Bias of Included Studies}

The majority of domains captured by the SYRCLE analysis were assessed as being unclear risk of bias for all studies (Suppl Table 2). All studies had an unclear risk of bias for sequence generation, as four studies [40, 45, 48, 49] did not mention randomisation while six studies were randomised but did not mention their method of randomisation. It was unclear whether baseline characteristics were balanced or accounted for in any of the studies, and it was unclear if the allocation of study animals was concealed in any of the studies. In addition, random housing and blinding of the intervention was not mentioned across all studies. None of the studies provided sufficient information to determine if random outcome assessment was consistently applied, with four studies mentioning random outcome assessment for histological outcomes only $[41,44,47,49]$. None of the studies provided sufficient information to determine if blinding of outcome assessors was consistent, with two studies mentioning blinding of assessors for histological outcomes only [45, 48]. It was unclear if any of the studies selectively reported their data, as none of the studies had a registered or posted a priori protocol.

\section{Discussion}

Our systematic review and meta-analysis highlights the therapeutic efficacy of MSC-EVs in pre-clinical models of diabetic wounds. MSC-EVs significantly improved wound closure, and these results were further enhanced with the use of MSCEVs enriched for specific non-coding RNAs and microRNAs. In addition, MSC-EVs increased blood vessel proliferation, re-epithelialization, and collagen deposition as well as decreased scarring and inflammation. Considering the high prevalence and treatment-resistant nature of diabetic wounds, the use of MSC-EVs appears highly encouraging. Future studies should leverage insights gained from our analysis to design definitive preclinical studies, which should accelerate the progression towards clinical studies.

Our observations in diabetic wounds are consistent with previous studies conducted on the efficacy of MSCs and MSC-EVs in the broader context of wound healing. In a recently published scoping review, we identified that MSC-EVs were beneficial across a variety of disease models in preclinical studies, including wound healing [32]. Specifically, several pre-clinical studies of MSC-EVs in non-diabetic models of acute and chronic wounds demonstrated the benefit of MSC-EVs, with minimal adverse events [50-52], however, no meta-analysis or pooled estimates of efficacy have been reported, to the best of our knowledge.

There have been relatively few clinical studies of MSC-EVs in patients, as most investigational products are not yet approved for human use. However, a prospective, nonrandomised, open- 


\begin{tabular}{|c|c|c|c|c|c|c|c|}
\hline \multirow[b]{2}{*}{ Study or Subgroup } & \multicolumn{3}{|c|}{ MSC-EVs } & \multicolumn{3}{|c|}{ Control } & \\
\hline & Mean & SD & Total & Mean & SD & Total & Weight \\
\hline Bian et al, 2020 & 53.4 & 6.9 & 5 & 30.1 & 4 & 5 & $10.9 \%$ \\
\hline Dalirfardouei et al, 2019 & 92 & 2.5 & 6 & 48 & 8 & 6 & $9.6 \%$ \\
\hline Ding et al, 2019 & 81.6 & 3.3 & 6 & 70.2 & 2.2 & 3 & $10.8 \%$ \\
\hline Ding et al, 2019 DFO & 91.2 & 1.8 & 6 & 70.2 & 4.4 & 3 & $8.6 \%$ \\
\hline Li et al, 2016 & 92.9 & 1.4 & 6 & 62.8 & 3.7 & 6 & $7.8 \%$ \\
\hline Li et al, 2019 & 46 & 5.8 & 12 & 41.7 & 4.5 & 6 & $12.1 \%$ \\
\hline Li et al, 2019 Edited & 82.4 & 1.4 & 12 & 61 & 2.9 & 6 & $9.1 \%$ \\
\hline Shi et al, 2017 & 56.9 & 2.8 & 4 & 37.8 & 3.6 & 4 & $9.3 \%$ \\
\hline Tao et al, 2016 & 96.7 & 2.6 & 6 & 73.6 & 1.9 & 6 & $8.1 \%$ \\
\hline Wang et al, 2019 & 44.7 & 4.7 & 3 & 7.6 & 5.9 & 3 & $7.1 \%$ \\
\hline Wang et al, 2019 Hydrogel & 63.9 & 8.3 & 3 & 7.6 & 5.9 & 3 & $6.5 \%$ \\
\hline Total $(95 \% \mathrm{Cl})$ & & & 69 & & & 51 & $100.0 \%$ \\
\hline
\end{tabular}

Fig. 2 Forest plot demonstrating increased wound closure rates of diabetic wounds receiving MSC-EVs. Note Li et al. 2019 used both unedited MSC-EVs and MSC-EVs from miR-126-3p overexpressing

label, cohort study in patients with COVID-19 infection [53], a smaller study in patients with acute kidney injury [54], and a case report of a patient with graft versus host disease after hematopoietic cell transplantation have reported beneficial outcomes with the use of MSC-EVs [55]. While the use of MSCEVs in patients with diabetic or non-diabetic wounds have not been reported, the clinical benefits and tolerability of MSCs have been reported across multiple studies. A systematic review [8] of nine clinical studies found adipose-derived MSCs to be effective and well-tolerated in the treatment of chronic ulcers and reduced wound-associated pain. Additionally, a randomised clinical trial [25] reported that MSCs derived from Wharton's jelly lead to accelerated wound healing in diabetic wounds, and another clinical study showed that MSCs promoted wound healing regardless of route of administration [6]. Taken together, MSCs and MSC-EVs appear well-tolerated and beneficial across a range of disease conditions, particularly in the setting of wound healing.
Std. Mean Difference

, Random, 95\% Cl

$3.73[1.29,6.17]$

$6.85[3.32,10.38]$

$3.35[0.86,5.83]$

$6.66[2.33,11.00]$

$9.93[4.95,14.91]$

$0.75[-0.26,1.77]$

$10.22[6.32,14.12]$

$5.15[1.35,8.95]$

$9.36[4.66,14.07]$

$5.56[-0.04,11.17]$

$6.25[0.01,12.50]$

5.84 [3.55, 8.13]

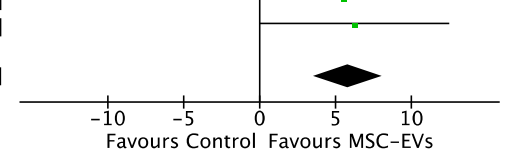

MSCs. DFO = deferoxamine. Control groups were inactive (no treatment or saline) except for 3 studies which used hydrogels without MSC-EVs as control (Li M 2016; Shi 2017; Tao 2016)

MSC-EVs are thought to promote wound healing through several mechanisms, mainly through immunomodulation and the promotion of angiogenesis $[15,56]$. Previous studies have shown that high glucose levels in diabetic mouse models enhance unrestrained M1 (pro-inflammatory) macrophage polarisation, inhibits M2 (anti-inflammatory) macrophage polarisation, and, subsequently, leads to the release of proinflammatory mediators, which drives chronic inflammation. This chronic inflammation leads to the destruction surrounding tissues and the establishment of chronic wounds. In studies identified in our analysis, MSC-EVs were reported to promote macrophage transition from M1 to M2 polarisation through decreased iNOS activity and increased ARG:iNOS ratio, which leads to increased levels of IL-10 and decreased levels of inflammatory markers IL-1 $\beta$ and TNF- $\alpha$. These results suggest that MSC-EVs have immunogenic properties to reduce inflammation and improve diabetic wound healing. MSC-EVs also promoted the growth of blood vessels, as both blood vessel

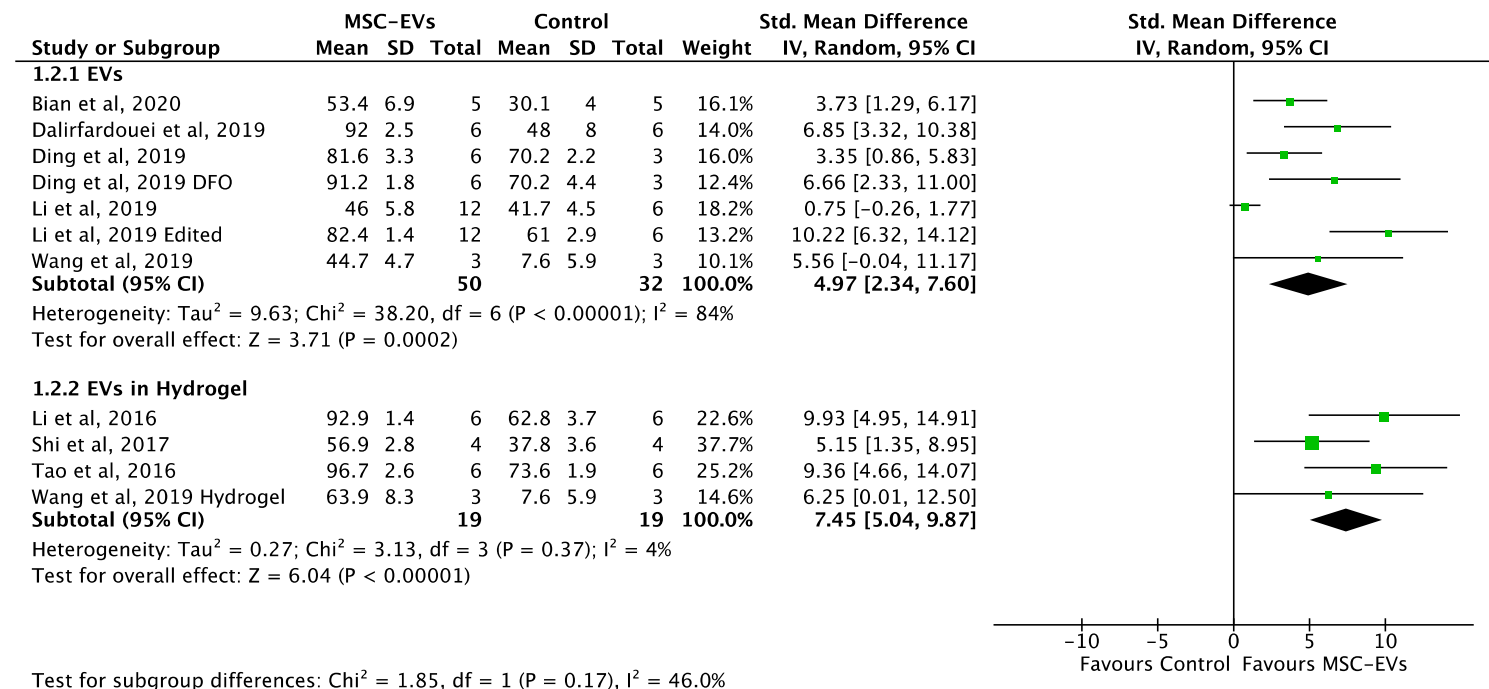

Fig. 3 Forest plot comparing wound closure rates of diabetic wounds receiving MSC-EVs delivered in conjunction with or without a hydrogel. $\mathrm{DFO}=$ deferoxamine. Control groups were inactive (no

treatment or saline) except for 3 studies which used hydrogels without MSC-EVs as control (Li M 2016; Shi 2017; Tao 2016) 


\begin{tabular}{|c|c|c|c|c|c|c|c|}
\hline \multirow[b]{2}{*}{ Study or Subgroup } & \multicolumn{3}{|c|}{ MSC-EVs } & \multicolumn{3}{|c|}{ Control } & \multirow[b]{2}{*}{ Weight } \\
\hline & Mean & SD & Total & Mean & SD & Total & \\
\hline \multicolumn{8}{|l|}{ 1.1.1 EVs } \\
\hline Bian et al, 2020 & 53.4 & 6.9 & 5 & 30.1 & 4 & 5 & $15.6 \%$ \\
\hline Dalirfardouei et al, 2019 & 92 & 2.5 & 6 & 48 & 8 & 6 & $12.5 \%$ \\
\hline Ding et al, 2019 & 81.6 & 3.3 & 6 & 70.2 & 2.2 & 3 & $15.4 \%$ \\
\hline Ding et al, 2019 DFO & 91.2 & 1.8 & 6 & 70.2 & 4.4 & 3 & $10.6 \%$ \\
\hline Li et al, 2019 & 46 & 5.8 & 12 & 41.7 & 4.5 & 6 & $19.1 \%$ \\
\hline Shi et al, 2017 & 56.9 & 2.8 & 4 & 37.8 & 3.6 & 4 & $11.8 \%$ \\
\hline Wang et al, 2019 & 44.7 & 4.7 & 3 & 7.6 & 5.9 & 3 & $8.0 \%$ \\
\hline $\begin{array}{l}\text { Wang et al, } 2019 \text { Hydrogel } \\
\text { Subtotal }(95 \% \mathrm{Cl})\end{array}$ & 63.9 & 8.3 & $\begin{array}{r}3 \\
45\end{array}$ & 7.6 & 5.9 & $\begin{array}{r}3 \\
33\end{array}$ & $\begin{array}{r}7.0 \% \\
100.0 \%\end{array}$ \\
\hline \multicolumn{8}{|c|}{$\begin{array}{l}\text { Heterogeneity: } \mathrm{Tau}^{2}=5.46 ; \mathrm{Chi}^{2}=26.11, \mathrm{df}=7(\mathrm{P}=0.0005) ; \mathrm{I}^{2}=73 \% \\
\text { Test for overall effect: } \mathrm{Z}=4.11(\mathrm{P}<0.0001)\end{array}$} \\
\hline \multicolumn{8}{|l|}{ 1.1.2 miRNA enriched EVs } \\
\hline Li et al, 2016 & 92.9 & 1.4 & 6 & 62.8 & 3.7 & 6 & $26.7 \%$ \\
\hline Li et al, 2019 & 82.4 & 1.4 & 12 & 61 & 2.9 & 6 & $43.5 \%$ \\
\hline $\begin{array}{l}\text { Tao et al, } 2016 \\
\text { Subtotal }(95 \% \mathrm{CI})\end{array}$ & 96.7 & 2.6 & $\begin{array}{r}6 \\
24\end{array}$ & 73.6 & 1.9 & $\begin{array}{r}6 \\
18\end{array}$ & $\begin{array}{r}29.8 \% \\
100.0 \%\end{array}$ \\
\hline
\end{tabular}

Test for subgroup differences: $\mathrm{Chi}^{2}=11.11, \mathrm{df}=1(\mathrm{P}=0.0009), \mathrm{I}^{2}=91.0 \%$

Fig. 4 Forest plot demonstrating increased wound closure rates of diabetic wounds receiving genetically modified MSC-EVs. DFO= deferoxamine. Control groups were inactive (no treatment or saline)

density and number were increased in diabetic wounds treated with MSC-EVs. Diabetic wounds have also been associated with reduced levels of VEGFa expression, resulting in impaired angiogenesis and slower healing [41] and MSC therapy can promote angiogenesis and increase VEGFa expression [57]. Studies in this systematic review show that VEGFa levels were increased in diabetic wounds treated with unmodified MSC$\mathrm{EVs}$, and angiogenic factors VEGF, TGF- $\beta 1$, and $\alpha$-SMA were further augmented after administration with MSC-EVs overexpressing long non-coding RNA H19. Overall, as noted in previous studies [32], these findings suggest that MSC-EVs have immunogenic and angiogenic properties, resulting in improved wound healing across studies.

This study has limitations worth mentioning. First, included studies had potential bias in many study design categories outlined by the SYRCLE Risk of Bias tool. Future studies can address this limitation by applying and reporting randomization, blinding, and a priori protocols. Second, a limited number of studies were identified for specific subgroup analyses which likely impact the power to detect efficacy, including studies that addressed the use of MSC-EVs in hydrogels. Third, murine models of streptozotocin-induced diabetes likely do not manifest the complete physiologic changes that occur in clinical Type I or Type II diabetes. However, this is a frequent and inherent limitation of pre-clinical models of disease. Furthermore, differences between studies were observed with regard to aspects of study design, including tissue source for derivation of MSCs and the dosing MSC-EVs, which likely prevented a more precise estimate of effect of MSC-EVs on wound closure. Despite heterogeneity across studies, consistent positive effects of MSC-EVs were observed which should buoy clinical translation efforts. While it is possible that our search failed to identify some studies that were overlooked,
Std. Mean Difference Std. Mean Difference IV, Random, $95 \% \mathrm{CI}$

$3.73[1.29,6.17]$

$6.85[3.32,10.38]$

$3.35[0.86,5.83]$

$6.66[2.33,11.00]$

$0.75[-0.26,1.77]$

$5.15[1.35,8.95]$

$5.56[-0.04,11.17]$

$6.25[0.01,12.50]$

$4.30[2.25,6.35]$

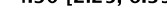
$9.93[4.95,14.91]$
$10.22[6.32,14.12]$
$9.36[4.66,14.07]$
$\mathbf{9 . 8 9}[\mathbf{7 . 3 2}, \mathbf{1 2 . 4 6}]$

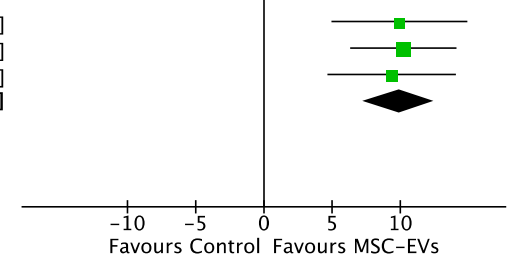

except for 3 studies which used hydrogels without MSC-EVs as control (Li M 2016; Shi 2017; Tao 2016)

we adopted robust systematic review methodology that minimizes this possibility.

In conclusion, MSC-EVs displayed substantial wound healing properties across a variety of outcomes and study designs in preclinical studies. MSC-EVs, and particularly modified MSC-EVs enriched for specific non-coding and microRNAs, are a promising treatment for diabetic wounds and warrant further investigation. Future studies should leverage insights gained from our analysis, such as reducing potential risks of bias and aligning outcome reporting to propel studies towards definitive pre-clinical and initial clinical studies.

Supplementary Information The online version contains supplementary material available at https://doi.org/10.1007/s12015-021-10164-4.

\section{Availability of Data and Materials N/A}

Authors Contributions CRediT Statement: Conceptualization: [Adrian Bailey, David Allan]; Methodology: [Adrian Bailey, David Allan]; Software: [Not applicable]; Validation: [Adrian Bailey, Heidi Li, Aidan Kirkham, David Allan], Formal analysis and investigation: [Adrian Bailey, Heidi Li, Aidan Kirkham, Harinard Magnati, David Allan]; Resources: [Heidi Elomazzen, David Allan]; Data curation: [Adrian Bailey, Heidi Li, Alvin Tieu, David Allan]; Writing - original draft preparation: [Adrian Bailey, Heidi Li, David Allan] Writing - review and editing: [Adrian Bailey, Heidi Li, Aidan Kirkham, Harinard Magnati, Alvin Tieu, Manoj Lalu, Dean Fergusson, Heidi Elomazzen, David Allan]; Visualization [Adrian Bailey, Heidi Li, Aidan Kirkham, Harinard Magnati, Alvin Tieu, Manoj Lalu, Heidi Elomazzen, David Allan]; Supervision: [Manoj Lalu, Dean Fergusson, Heidi Elomazzen, David Allan]; Project Administration: [Manoj Lalu, Dean Fergusson, Heidi Elomazzen, David Allan] Funding acquisition: [Heidi Elomazzen, David Allan].

Funding Canadian Blood Services provided stipend support for $\mathrm{AB}$ and AK. MML is supported by The Ottawa Hospital Anesthesia Alternate 
Funds Association and holds a University of Ottawa Junior Research Chair in Innovative Translational Research.

\section{Declarations}

Competing Interests HE and DSA are paid consultants and/or employees of Canadian Blood Services.

Ethical Approval This study met the definition of Institutional Review Board exempt research.

\section{Consent to Participate N/A}

Consent to Publish N/A

\section{References}

1. Fard, A. S., Esmaelzadeh, M., \& Larijani, B. (2007 Nov). Assessment and treatment of diabetic foot ulcer. International Journal of Clinical Practice, 61(11), 1931-1938.

2. Yazdanpanah, L., Nasiri, M., \& Adarvishi, S. (2015 Feb). Literature review on the management of diabetic foot ulcer. World Journal of Diabetes, 6(1), 37-53.

3. Armstrong, D. G., Boulton, A. J. M., \& Bus, S. A. (2017 Jun). Diabetic foot ulcers and their recurrence. The New England Journal of Medicine, 376(24), 2367-2375.

4. Shaw, J. E., Sicree, R. A., \& Zimmet, P. Z. (2010 Jan). Global estimates of the prevalence of diabetes for 2010 and 2030. Diabetes Research and Clinical Practice, 87(1), 4-14.

5. Yager, D. R., Kulina, R. A., \& Gilman, L. A. (2007 Dec). Wound fluids: A window into the wound environment? The International Journal of Lower Extremity Wounds, 6(4), 262-272.

6. Amato, B., Compagna, R., Amato, M., Butrico, L., Fugetto, F., Chibireva, M. D., Barbetta, A., Cannistrà, M., de Franciscis, S., \& Serra, R. (2016). The role of adult tissue-derived stem cells in chronic leg ulcers: A systematic review focused on tissue regeneration medicine. International Wound Journal, 13(5), 1289-1298.

7. Wu, Y., Chen, L., Scott, P. G., \& Tredget, E. E. (2007). Mesenchymal stem cells enhance wound healing through differentiation and angiogenesis. Stem Cells, 25(10), 2648-2659.

8. Holm, J. S., Toyserkani, N. M., \& Sorensen, J. A. (2018). Adiposederived stem cells for treatment of chronic ulcers: Current status. Stem Cell Research \& Therapy, 9(1), 142.

9. Gao, F., Chiu, S. M., Motan, D. A. L., Zhang, Z., Chen, L., Ji, H. L., Tse, H. F., Fu, Q. L., \& Lian, Q. (2016). Mesenchymal stem cells and immunomodulation: Current status and future prospects. Cell Death \& Disease, 7(1), e2062.

10. Shabbir, A., Cox, A., Rodriguez-Menocal, L., Salgado, M., \& Van Badiavas, E. (2015). Mesenchymal stem cell Exosomes induce proliferation and migration of Normal and chronic wound fibroblasts, and enhance angiogenesis in vitro. Stem Cells Dev [Internet]., 24(14), 1635-1647 Available from: http://ovidsp.ovid.com/ ovidweb.cgi? $\mathrm{T}=\mathrm{JS} \& \mathrm{PAGE}=$ reference $\& \mathrm{D}=\operatorname{med} 12 \& \mathrm{NEWS}=$ $\mathrm{N} \& A N=25867197$.

11. Mohajer Ansari, J., Ramhormozi, P., Shabani, R., Pazoki-Toroudi, H., Yari, A., Barati, M., Dahmardehei, M., Babakhani, A., \& Nobakht, M. (2020 Jun). Simvastatin combined with bone marrow mesenchymal stromal cells (BMSCs) improve burn wound healing by ameliorating angiogenesis through SDF- $1 \alpha / \mathrm{CXCR} 4$ pathway. Iranian Journal of Basic Medical Sciences, 23(6), 751-759.

12. S.-I. M. Mesenchymal stem cells: The roles and functions in cutaneous wound healing and tumor growth. J Dermatol Sci [Internet].
2017;86(2):83-9. Available from: http://www.elsevier.com/locate/ jdermsci

13. Ahmadi, A. R., Chicco, M., Huang, J., Qi, L., Burdick, J., Williams, G. M., Cameron, A. M., \& Sun, Z. (2019). Stem cells in burn wound healing: A systematic review of the literature. Burns., 45(5), 1014-1023.

14. P. G. Concise review: Adipose-derived stem cells (ASCs) and adipocyte-secreted exosomal microrna (A-SE-miR) modulate cancer growth and promote wound repair. J Clin Med [Internet]. 2019;8(6):855. Available from: https://www.mdpi.com/20770383/8/6/855/pdf

15. An, T., Chen, Y., Tu, Y., Lin, P. (2021). Mesenchymal stromal cellderived extracellular vesicles in the treatment of diabetic foot ulcers: Application and challenges. Stem Cell Reviews and Reports, 17(2), 369-378. https://doi.org/10.1007/s12015-020-10014-9.

16. Prakoeswa, C. R. S., Natallya, F. R., Harnindya, D., Thohiroh, A., Oktaviyanti, R. N., Pratiwi, K. D., Rubianti, M. A., Yogatri, B., Primasari, P. I., Herwanto, N., Alinda, M. D., Kusumaputra, B. H., Astari, L., Listiawan, M. Y., Agusni, I., \& Rantam, F. A. (2018). The efficacy of topical human amniotic membrane-mesenchymal stem cell-conditioned medium (hAMMSC-CM) and a mixture of topical hAMMSC-CM + vitamin C and hAMMSC-CM + vitamin $\mathrm{E}$ on chronic plantar ulcers in leprosy:A randomized control trial. The Journal of Dermatological Treatment, 29(8), 835-840.

17. Wang, L., Wang, L., Cong, X., Liu, G., Zhou, J., Bai, B., Li, Y., Bai, W., Li, M., Ji, H., Zhu, D., Wu, M., \& Liu, Y. (2013). Human umbilical cord mesenchymal stem cell therapy for patients with active rheumatoid arthritis: Safety and efficacy. Stem Cells and Development, 22(24), 3192-3202.

18. Ciccocioppo, R., Bernardo, M. E., Sgarella, A., Maccario, R., Avanzini, M. A., Ubezio, C., Minelli, A., Alvisi, C., Vanoli, A., Calliada, F., Dionigi, P., Perotti, C., Locatelli, F., \& Corazza, G. R. (2011). Autologous bone marrow-derived mesenchymal stromal cells in the treatment of fistulising Crohn's disease. Gut., 60(6), 788-798.

19. Zhao, L., Chen, S., Yang, P., Cao, H., \& Li, L. (2019 Jun). The role of mesenchymal stem cells in hematopoietic stem cell transplantation: Prevention and treatment of graft-versus-host disease. Stem Cell Research \& Therapy, 10(1), 182.

20. Kim, H.-S., Lee, J. H., Roh, K.-H., Jun, H. J., Kang, K.-S., \& Kim, T.-Y. (2017 Jan). Clinical trial of human umbilical cord bloodderived stem cells for the treatment of moderate-to-severe atopic dermatitis: Phase I/IIa studies. Stem Cells, 35(1), 248-255.

21. Leyendecker, A. J., Pinheiro, C. C. G., Amano, M. T., \& Bueno, D. F. (2018). The use of human Mesenchymal stem cells as therapeutic agents for the in vivo treatment of immune-related diseases: A systematic review. Frontiers in Immunology, 9, 2056.

22. Lalu, M. M., McIntyre, L., Pugliese, C., Fergusson, D., Winston, B. W., Marshall, J. C., Granton, J., Stewart, D. J., \& Canadian Critical Care Trials Group. (2012). Safety of cell therapy with mesenchymal stromal cells (SafeCell): A systematic review and meta-analysis of clinical trials. PLoS One, 7(10), e47559.

23. Rodríguez-Fuentes, D. E., Fernández-Garza, L. E., Samia-Meza, J. A., Barrera-Barrera, S. A., Caplan, A. I., \& Barrera-Saldaña, H. A. (2021 Jan). Mesenchymal stem cells current clinical applications: A systematic review. Archives of Medical Research, 52(1), 93-101.

24. Carvello, M., Lightner, A., Yamamoto, T., Kotze, P. G., \& Spinelli, A. (2019). Mesenchymal stem cells for perianal Crohn's disease. Cells., 8(7), 764.

25. Hashemi, S.-S., Mohammadi, A. A., Kabiri, H., Hashempoor, M. R., Mahmoodi, M., Amini, M., \& Mehrabani, D. (2019 Dec). The healing effect of Wharton's jelly stem cells seeded on biological scaffold in chronic skin ulcers: A randomized clinical trial. Journal of Cosmetic Dermatology, 18(6), 1961-1967.

26. Moon, K. C., Suh, H. S., Kim, K. B., Han, S. K., Young, K. W., Lee, J. W., \& Kim, M. H. (2019). Potential of allogeneic adipose- 
derived stem cell-hydrogel complex for treating diabetic foot ulcers. Diabetes., 68(4), 837-846.

27. Théry, C., Witwer, K. W., Aikawa, E., Alcaraz, M. J., Anderson, J. D., Andriantsitohaina, R., Antoniou, A., Arab, T., Archer, F., Atkin-Smith, G. K., Ayre, D. C., Bach, J. M., Bachurski, D., Baharvand, H., Balaj, L., Baldacchino, S., Bauer, N. N., Baxter, A. A., Bebawy, M., Beckham, C., Bedina Zavec, A., Benmoussa, A., Berardi, A. C., Bergese, P., Bielska, E., Blenkiron, C., BobisWozowicz, S., Boilard, E., Boireau, W., Bongiovanni, A., Borràs, F. E., Bosch, S., Boulanger, C. M., Breakefield, X., Breglio, A. M., Brennan, M. Á., Brigstock, D. R., Brisson, A., Broekman, M. L. D., Bromberg, J. F., Bryl-Górecka, P., Buch, S., Buck, A. H., Burger, D., Busatto, S., Buschmann, D., Bussolati, B., Buzás, E. I., Byrd, J. B., Camussi, G., Carter, D. R. F., Caruso, S., Chamley, L. W., Chang, Y. T., Chen, C., Chen, S., Cheng, L., Chin, A. R., Clayton, A., Clerici, S. P., Cocks, A., Cocucci, E., Coffey, R. J., Cordeiro-da-Silva, A., Couch, Y., Coumans, F. A. W., Coyle, B., Crescitelli, R., Criado, M. F., D’Souza-Schorey, C., Das, S., Datta Chaudhuri, A., de Candia, P., de Santana Jr., E. F., de Wever, O., del Portillo, H. A., Demaret, T., Deville, S., Devitt, A., Dhondt, B., di Vizio, D., Dieterich, L. C., Dolo, V., Dominguez Rubio, A. P., Dominici, M., Dourado, M. R., Driedonks, T. A. P., Duarte, F. V., Duncan, H. M., Eichenberger, R. M., Ekström, K., el Andaloussi, S., Elie-Caille, C., Erdbrügger, U., Falcón-Pérez, J. M., Fatima, F., Fish, J. E., Flores-Bellver, M., Försönits, A., Frelet-Barrand, A., Fricke, F., Fuhrmann, G., Gabrielsson, S., Gámez-Valero, A., Gardiner, C., Gärtner, K., Gaudin, R., Gho, Y. S., Giebel, B., Gilbert, C., Gimona, M., Giusti, I., Goberdhan, D. C. I., Görgens, A., Gorski, S. M., Greening, D. W., Gross, J. C., Gualerzi, A., Gupta, G. N., Gustafson, D., Handberg, A., Haraszti, R. A., Harrison, P., Hegyesi, H., Hendrix, A., Hill, A. F., Hochberg, F. H., Hoffmann, K. F., Holder, B., Holthofer, H., Hosseinkhani, B., Hu, G., Huang, Y., Huber, V., Hunt, S., Ibrahim, A. G. E., Ikezu, T., Inal, J. M., Isin, M., Ivanova, A., Jackson, H. K., Jacobsen, S., Jay, S. M., Jayachandran, M., Jenster, G., Jiang, L., Johnson, S. M., Jones, J. C., Jong, A., Jovanovic-Talisman, T., Jung, S., Kalluri, R., Kano, S. I., Kaur, S., Kawamura, Y., Keller, E. T., Khamari, D., Khomyakova, E., Khvorova, A., Kierulf, P., Kim, K. P., Kislinger, T., Klingeborn, M., Klinke II, D. J., Kornek, M., Kosanović, M. M., Kovács, Á. F., Krämer-Albers, E. M., Krasemann, S., Krause, M., Kurochkin, I. V., Kusuma, G. D., Kuypers, S., Laitinen, S., Langevin, S. M., Languino, L. R., Lannigan, J., Lässer, C., Laurent, L. C., Lavieu, G., Lázaro-Ibáñez, E., le Lay, S., Lee, M. S., Lee, Y. X. F., Lemos, D. S., Lenassi, M., Leszczynska, A., Li, I. T. S., Liao, K., Libregts, S. F., Ligeti, E., Lim, R., Lim, S. K., Linē, A., Linnemannstöns, K., Llorente, A., Lombard, C. A., Lorenowicz, M. J., Lörincz, Á. M., Lötvall, J., Lovett, J., Lowry, M. C., Loyer, X., Lu, Q., Lukomska, B., Lunavat, T. R., Maas, S. L. N., Malhi, H., Marcilla, A., Mariani, J., Mariscal, J., MartensUzunova, E. S., Martin-Jaular, L., Martinez, M. C., Martins, V. R., Mathieu, M., Mathivanan, S., Maugeri, M., McGinnis, L. K., McVey, M. J., Meckes Jr., D. G., Meehan, K. L., Mertens, I., Minciacchi, V. R., Möller, A., Møller Jørgensen, M., MoralesKastresana, A., Morhayim, J., Mullier, F., Muraca, M., Musante, L., Mussack, V., Muth, D. C., Myburgh, K. H., Najrana, T., Nawaz, M., Nazarenko, I., Nejsum, P., Neri, C., Neri, T., Nieuwland, R., Nimrichter, L., Nolan, J. P., Nolte-'t Hoen, E. N. M., Noren Hooten, N., O’Driscoll, L., O’Grady, T., O'Loghlen, A., Ochiya, T., Olivier, M., Ortiz, A., Ortiz, L. A., Osteikoetxea, X., Østergaard, O., Ostrowski, M., Park, J., Pegtel, D. M., Peinado, H., Perut, F., Pfaffl, M. W., Phinney, D. G., Pieters, B. C. H., Pink, R. C., Pisetsky, D. S., Pogge von Strandmann, E., Polakovicova, I., Poon, I. K. H., Powell, B. H., Prada, I., Pulliam, L., Quesenberry, P., Radeghieri, A., Raffai, R. L., Raimondo, S., Rak, J., Ramirez, M. I., Raposo, G., Rayyan, M. S., Regev-Rudzki, N., Ricklefs, F. L., Robbins, P. D., Roberts, D. D., Rodrigues, S. C., Rohde, E.,
Rome, S., Rouschop, K. M. A., Rughetti, A., Russell, A. E., Saá, P., Sahoo, S., Salas-Huenuleo, E., Sánchez, C., Saugstad, J. A., Saul, M. J., Schiffelers, R. M., Schneider, R., Schøyen, T. H., Scott, A., Shahaj, E., Sharma, S., Shatnyeva, O., Shekari, F., Shelke, G. V., Shetty, A. K., Shiba, K., Siljander, P. R. M., Silva, A. M., Skowronek, A., Snyder II, O. L., Soares, R. P., Sódar, B. W., Soekmadji, C., Sotillo, J., Stahl, P. D., Stoorvogel, W., Stott, S. L., Strasser, E. F., Swift, S., Tahara, H., Tewari, M., Timms, K., Tiwari, S., Tixeira, R., Tkach, M., Toh, W. S., Tomasini, R., Torrecilhas, A. C., Tosar, J. P., Toxavidis, V., Urbanelli, L., Vader, P., van Balkom, B. W. M., van der Grein, S. G., van Deun, J., van Herwijnen, M. J. C., van Keuren-Jensen, K., van Niel, G., van Royen, M. E., van Wijnen, A. J., Vasconcelos, M. H., Vechetti Jr., I. J., Veit, T. D., Vella, L. J., Velot, É., Verweij, F. J., Vestad, B., Viñas, J. L., Visnovitz, T., Vukman, K. V., Wahlgren, J., Watson, D. C., Wauben, M. H. M., Weaver, A., Webber, J. P., Weber, V., Wehman, A. M., Weiss, D. J., Welsh, J. A., Wendt, S., Wheelock, A. M., Wiener, Z., Witte, L., Wolfram, J., Xagorari, A., Xander, P., Xu, J., Yan, X., Yáñez-Mó, M., Yin, H., Yuana, Y., Zappulli, V., Zarubova, J., Žèkas, V., Zhang, J. Y., Zhao, Z., Zheng, L., Zheutlin, A. R., Zickler, A. M., Zimmermann, P., Zivkovic, A. M., Zocco, D., \& Zuba-Surma, E. K. (2018). Minimal information for studies of extracellular vesicles 2018 (MISEV2018): A position statement of the International Society for Extracellular Vesicles and update of the MISEV2014 guidelines. J Extracell Vesicles., 7(1), 1535750.

28. Casado-Diaz, A., Quesada-Gomez, J. M., \& Dorado, G. (2020). Extracellular Vesicles Derived From Mesenchymal Stem Cells (MSC) in Regenerative Medicine: Applications in Skin Wound Healing. Front Bioeng Biotechnol [Internet], 8, 146 Available from: http://ovidsp.ovid.com/ovidweb.cgi?T=JS\&PAGE= reference $\& D=$ prem\&NEWS $=\mathrm{N} \& A N=32195233$.

29. Ti, D., Hao, H., Fu, X., \& Han, W. (2016). Mesenchymal stem cells-derived exosomal microRNAs contribute to wound inflammation. Science China. Life Sciences, 59(12), 1305-1312.

30. Tieu, A., Slobodian, M., Fergusson, D. A., Montroy, J., Burger, D., Stewart, D. J., Shorr, R., Allan, D. S., \& Lalu, M. M. (2019). Methods and efficacy of extracellular vesicles derived from mesenchymal stromal cells in animal models of disease: A preclinical systematic review protocol. Systematic Reviews, 8(1), 322.

31. Allan, D., Tieu, A., Lalu, M., \& Burger, D. (2020). Mesenchymal stromal cell-derived extracellular vesicles for regenerative therapy and immune modulation: Progress and challenges toward clinical application. Stem Cells Translational Medicine, 9(1), 39-46.

32. Tieu, A., Lalu, M. M., Slobodian, M., Gnyra, C., Fergusson, D. A., Montroy, J., Burger, D., Stewart, D. J., \& Allan, D. S. (2020 Aug). An analysis of Mesenchymal stem cell-derived extracellular vesicles for preclinical use. ACS Nano, 14(8), 9728-9743.

33. Moher, D., Liberati, A., Tetzlaff, J., Altman, D. G., Altman, D., Antes, G., et al. (2009). Preferred reporting items for systematic reviews and meta-analyses: The PRISMA statement. PLoS Medicine, 6(7), e1000097.

34. McGowan, J., Sampson, M., Salzwedel, D. M., Cogo, E., Foerster, V., \& Lefebvre, C. (2016). PRESS peer review of electronic search strategies: 2015 guideline statement. Journal of Clinical Epidemiology, 75, 40-46.

35. Dominici, M., Le Blanc, K., Mueller, I., Slaper-Cortenbach, I., Marini, F., Krause, D., et al. (2006). Minimal criteria for defining multipotent mesenchymal stromal cells. The International Society for Cellular Therapy position statement. Cytotherapy., 8(4), 315317.

36. Hooijmans, C. R., Rovers, M. M., De Vries, R. B. M., Leenaars, M., Ritskes-Hoitinga, M., \& Langendam, M. W. (2014). SYRCLE's risk of bias tool for animal studies. BMC Medical Research Methodology, 14, 43. 
37. The Nordic Cochrane Centre (2014) Review manager (RevMan). Cochrane Collab.

38. Cochrane Handbook for Systematic Reviews of Interventions. Cochrane Handbook for Systematic Reviews of Interventions. 2019.

39. Ripsman, D., Fergusson, D. A., Montroy, J., Auer, R. C., Huang, J. W., Dobriyal, A., Wesch, N., Carrier, M., \& Lalu, M. M. (2020 Jul). A systematic review on the efficacy and safety of low molecular weight heparin as an anticancer therapeutic in preclinical animal models. Thrombosis Research, 195, 103-113.

40. Wang, C., Wang, M., Xu, T., Zhang, X., Lin, C., Gao, W., Xu, H., Lei, B., \& Mao, C. (2019). Engineering bioactive self-healing antibacterial Exosomes hydrogel for promoting chronic diabetic wound healing and complete skin regeneration. Theranostics., 9(1), 65-76.

41. Bian, X., Li, B., Yang, J., Ma, K., Sun, M., Zhang, C., \& Fu, X. (2020 Apr). Regenerative and protective effects of dMSC-sEVs on high-glucose-induced senescent fibroblasts by suppressing RAGE pathway and activating Smad pathway. Stem Cell Research \& Therapy, 11(1), 166.

42. Dalirfardouei, R., Jamialahmadi, K., Jafarian, A. H., \& Mahdipour, E. (2019 Apr). Promising effects of exosomes isolated from menstrual blood-derived mesenchymal stem cell on wound-healing process in diabetic mouse model. Journal of Tissue Engineering and Regenerative Medicine, 13(4), 555-568.

43. Ding, J., Wang, X., Chen, B., Zhang, J., \& Xu, J. (2019). Exosomes derived from human bone marrow Mesenchymal stem cells stimulated by Deferoxamine accelerate cutaneous wound healing by promoting angiogenesis. BioMed Research International, 2019, 9742765 .

44. Li, B., Luan, S., Chen, J., Zhou, Y., Wang, T., Li, Z., Fu, Y., Zhai, A., \& Bi, C. (2020 Mar). The MSC-derived Exosomal lncRNA H19 promotes wound healing in diabetic foot ulcers by Upregulating PTEN via MicroRNA-152-3p. Mol Ther Nucleic Acids., 19, 814-826.

45. Li, M., Ke, Q.-F., Tao, S.-C., Guo, S.-C., Rui, B.-Y., \& Guo, Y.-P. (2016). Fabrication of hydroxyapatite/chitosan composite hydrogels loaded with exosomes derived from miR-126-3p overexpressed synovial mesenchymal stem cells for diabetic chronic wound healing. J Mater Chem B [Internet]., 4(42), 6830-6841 Available from: http://ovidsp.ovid.com/ovidweb.cgi?T= JS\&PAGE=reference \&D=prem\&NEWS=N\&AN=32263577.

46. Shi, R., Jin, Y., Hu, W., Lian, W., Cao, C., Han, S., Zhao, S., Yuan, H., Yang, X., Shi, J., \& Zhao, H. (2020). Exosomes derived from mmu_circ_0000250-modified adipose-derived mesenchymal stem cells promote wound healing in diabetic mice by inducing miR128-3p/SIRT1-mediated autophagy. Am J Physiol - Cell Physiol [Internet]., 318(5), C848-C856. https://doi.org/10.1152/ajpcell. 00041.2020

47. Shi, Q., Qian, Z., Liu, D., Sun, J., Wang, X., Liu, H., Xu, J., \& Guo, X. (2017). GMSC-derived Exosomes combined with a chitosan/ silk hydrogel sponge accelerates wound healing in a diabetic rat skin defect model. Frontiers in Physiology, 8, 904.

48. Tao, S.-C., Guo, S.-C., Li, M., Ke, Q.-F., Guo, Y.-P., \& Zhang, C.Q. (2017 Mar). Chitosan wound dressings incorporating Exosomes derived from MicroRNA-126-overexpressing Synovium Mesenchymal stem cells provide sustained release of Exosomes and heal full-thickness skin defects in a diabetic rat model. Stem Cells Translational Medicine, 6(3), 736-747.

49. Ti, D., Hao, H., Tong, C., Liu, J., Dong, L., Zheng, J., et al. (2015). LPS - preconditioned mesenchymal stromal cells modify macrophage polarization for resolution of chronic inflammation via exosome - shuttled let - 7b. Journal of Translational Medicine, $28,1-14$.

50. Hu, Y., Rao, S. S., Wang, Z. X., Cao, J., Tan, Y. J., Luo, J., Li, H. M., Zhang, W. S., Chen, C. Y., \& Xie, H. (2018). Exosomes from human umbilical cord blood accelerate cutaneous wound healing through miR-21-3p-mediated promotion of angiogenesis and fibroblast function. Theranostics., 8(1), 169-184.

51. Zhang, J., Guan, J., Niu, X., Hu, G., Guo, S., Li, Q., et al. (2015). Exosomes released from human induced pluripotent stem cellsderived MSCs facilitate cutaneous wound healing by promoting collagen synthesis and angiogenesis. Journal of Translational Medicine, 1(13), 49.

52. Chen, B., Sun, Y., Zhang, J., Zhu, Q., Yang, Y., Niu, X., Deng, Z., Li, Q., \& Wang, Y. (2019). Human embryonic stem cell-derived exosomes promote pressure ulcer healing in aged mice by rejuvenating senescent endothelial cells. Stem Cell Research \& Therapy, 10(1), 142.

53. Sengupta, V., Sengupta, S., Lazo, A., Woods, P., Nolan, A., \& Bremer, N. (2020 Jun). Exosomes derived from bone marrow Mesenchymal stem cells as treatment for severe COVID-19. Stem Cells and Development, 29(12), 747-754.

54. Nassar, W., El-Ansary, M., Sabry, D., Mostafa, M. A., Fayad, T., Kotb, E., et al. (2016). Umbilical cord mesenchymal stem cells derived extracellular vesicles can safely ameliorate the progression of chronic kidney diseases. Biomater Res., 20, 21.

55. Kordelas, L., Rebmann, V., Ludwig, A. K., Radtke, S., Ruesing, J., Doeppner, T. R., Epple, M., Horn, P. A., Beelen, D. W., \& Giebel, B. (2014). MSC-derived exosomes: A novel tool to treat therapyrefractory graft-versus-host disease. Leukemia., 28(4), 970-973.

56. Bernardo, M. E., \& Fibbe, W. E. (2013). Mesenchymal stromal cells: Sensors and switchers of inflammation. Cell Stem Cell, 13(4), 392-402.

57. Ha, D. H., Kim, H.-K., Lee, J., Kwon, H. H., Park, G.-H., Yang, S. H., et al. (2020). Mesenchymal stem/stromal cell-derived exosomes for immunomodulatory therapeutics and skin regeneration. Cells, 9(5), 1157. https://doi.org/10.3390/cells9051157.

Publisher's Note Springer Nature remains neutral with regard to jurisdictional claims in published maps and institutional affiliations. 